LAWRENCE LIVERMORE NATIONAL LABORATORY

\title{
Soil Composition, Area 6 of the Nevada National Security Site
}

\author{
K. B. Knight, R. Albo, G. Eppich, S. Roberts, M. Singleton \\ Lawrence Livermore National Laboratory
}

September 2012 


\section{Disclaimer}

This document was prepared as an account of work sponsored by an agency of the United States government. Neither the United States government nor Lawrence Livermore National Security, LLC, nor any of their employees makes any warranty, expressed or implied, or assumes any legal liability or responsibility for the accuracy, completeness, or usefulness of any information, apparatus, product, or process disclosed, or represents that its use would not infringe privately owned rights. Reference herein to any specific commercial product, process, or service by trade name, trademark, manufacturer, or otherwise does not necessarily constitute or imply its endorsement, recommendation, or favoring by the United States government or Lawrence Livermore National Security, LLC. The views and opinions of authors expressed herein do not necessarily state or reflect those of the United States government or Lawrence Livermore National Security, LLC, and shall not be used for advertising or product endorsement purposes.

\section{Auspices Statement}

This work performed under the auspices of the U.S. Department of Energy by Lawrence Livermore National Laboratory under Contract DE-AC52-07NA27344. 


\section{Soil Composition, Area 6}

Three soil samples (two from the surrounding soils and one from the intersecting gravel roadbed) were collected from Frenchman Flat Area 6, across from the DAF at the Nevada National Security Site for analysis of major and

\begin{tabular}{cl}
\multicolumn{2}{c}{ Table 1: Samples } \\
Sample & Origin \\
A & Area 6 soil \\
B & Area 6 soil \\
C & Area 6 roadbed
\end{tabular}
trace element compositions.

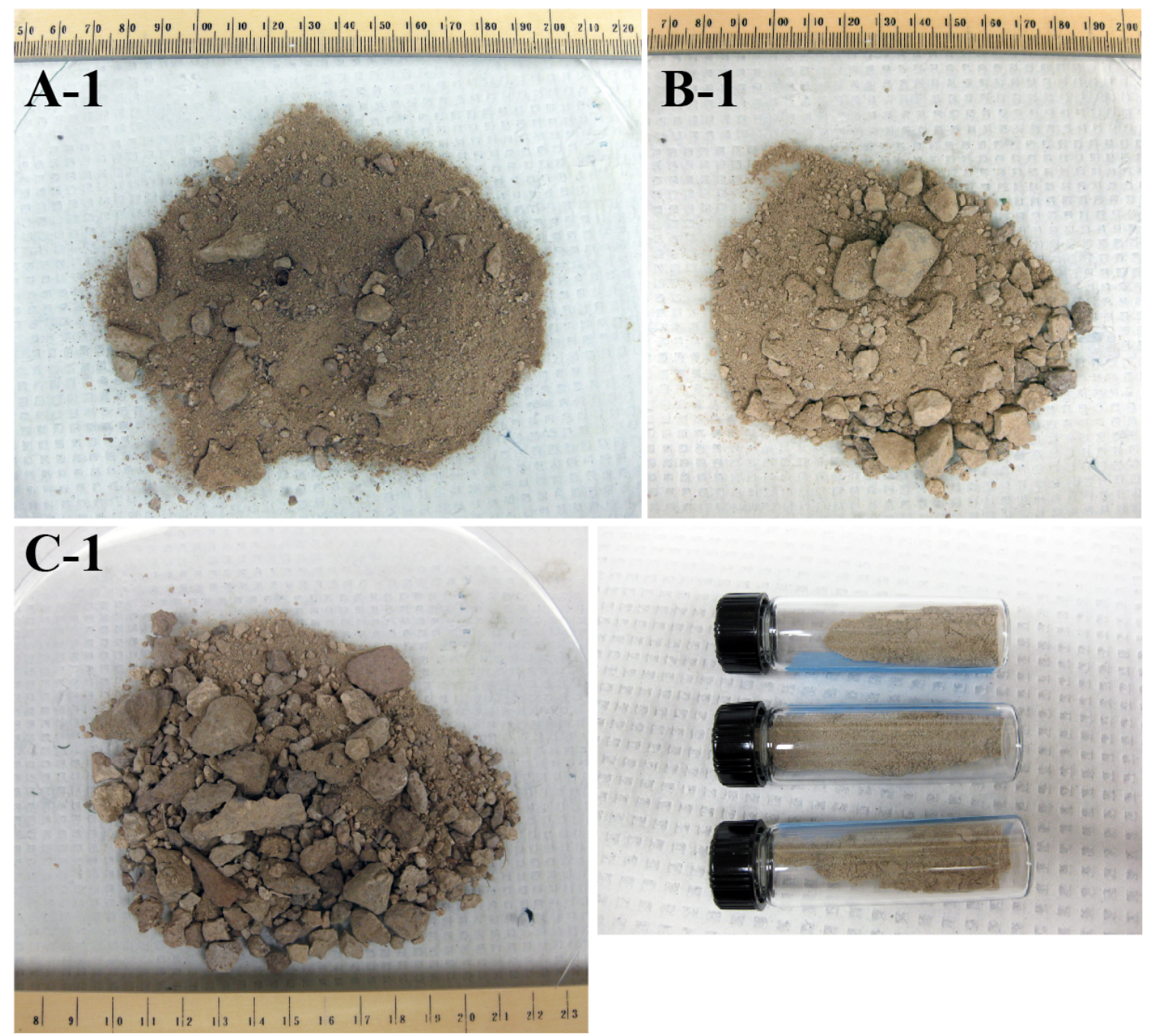

Figure 1: Raw (as provided) and crushed (vials) soil samples

Sub-samples were homogenized into a powder in an agate mortar. Aliquots were then distributed for various types of analysis. A summary of these analyses are given below: 
Table 2: Analytical Techniques

\section{Technique \\ Mineral Analysis by Powder Diffraction (XRD) \\ Light element combustion analysis \\ X-ray fluorescence (XRF) \\ Plasma Mass Spectrometry (ICP-MS)}

\section{Elements}

Major mineral phases

$\mathrm{C}, \mathrm{H}, \mathrm{N}, \mathrm{O}, \mathrm{S}$

Major and trace elements

Major and trace elements

\section{Mineral Analysis by Powder Diffraction}

X-ray powder diffraction (XRD) is a rapid analytical technique primarily used for phase identification of a crystalline material based on constructive interference of monochromatic X-rays and a crystalline sample. The analyzed material is finely ground, homogenized, and average bulk composition is determined. X-ray powder diffraction is most widely used for the identification of unknown crystalline materials (e.g. minerals, inorganic compounds).

For these soils, powdered samples were analyzed on a Bruker AXS D8 ADVANCE Xray diffractometer equipped with a LynxEye 1-dimentional linear $\mathrm{Si}$ strip detector. DIFFRACplus Evaluation package Release 2009 software was used for the data analysis. The unknown samples were scanned from $5-75^{\circ} 2 \theta$. The step scan parameters were $0.02^{\circ}$ step and 2 second counting time per step with a $15 \mathrm{~mm}$ variable divergence slit and a $1.0^{\circ}$ antiscatter slit. The samples were $\mathrm{x}$-rayed with $\mathrm{Ni}$-filter $\mathrm{Cu}$ radiation from a sealed tube operated at $40 \mathrm{kV}$ and $40 \mathrm{~mA}$. Phases in the unknown samples were identified by comparison of observed peaks to those in the International Centre for Diffraction Data (ICDD PDF2009) powder diffraction database. X-ray reference material (Bruker supplied $\mathrm{Al}_{2} \mathrm{O}_{3}$ ) was analyzed during the time of the unknown runs to ensure goniometer alignment. No peak shift was observed in the reference material.

Table 3: XRD results

$\begin{array}{cll}\text { Sample } & \text { Major phase } & \text { Minor phases } \\ \text { A1 } & \text { Quartz } & \text { Na-, Ca-, and K- feldspar, minor amounts of iron and clay } \\ \text { B1 } & \text { Quartz } & \text { Na-, Ca-, and K- feldspar, minor amounts of iron and clay } \\ \text { C1 } & \text { Quartz } & \text { Na-, Ca-, and K- feldspar, minor amounts of iron and clay }\end{array}$

XRD scans revealed similar mineralogy for all three samples (the two soils as well as the road bed sample), with quartz $\left(\mathrm{SiO}_{2}\right)$ as the major mineral phase. Feldspar minerals $\left(\mathrm{KAlSi}_{3} \mathrm{O}_{8}-\mathrm{NaAlSi}_{3} \mathrm{O}_{8}-\mathrm{CaAl}_{2} \mathrm{Si}_{2} \mathrm{O}_{8}\right)$ were the dominant minor constituents. Sample $\mathrm{C}$ contained significant Albite (Ca-feldspar) absent in the other two samples. 


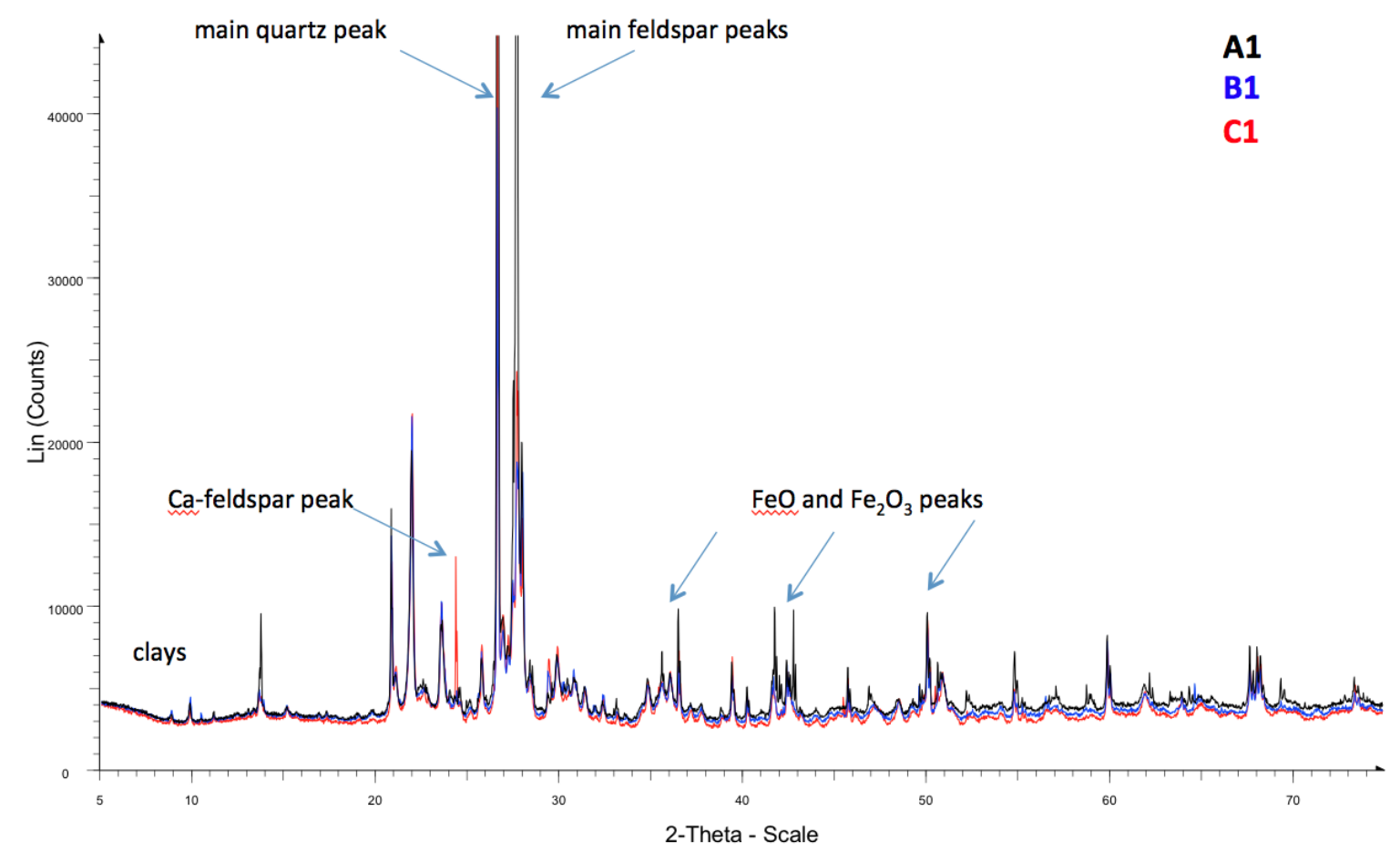

Figure 2: X-ray diffraction scans of the three samples (not background subtracted) showing the similarities in diffraction patterns and mineralogical composition.

\section{Light Element Combustion Analyses}

The carbon and nitrogen analysis was performed using the EA 1100 Elemental Analyzer (CE Instruments). This is an automated dry combustion system utilizing high-temperature combustion (oxidation) of the sample, followed by use of a carrier gas system to move the oxidized products through the system to the analyzer.

A sulfanilamide standard was used to prepare the linear calibration curve for the 2 elements. Approximately $20 \mathrm{mg}$ of soil from each sample was loaded into tin capsules for analysis, each soil analyzed in triplicate. Samples were combusted in a column held at $920^{\circ} \mathrm{C}$ consisting of $\mathrm{WO}_{3}$ and copper. When the sample is dropped into the combustion tube, the exothermic Sn combustion reaction reaches $1700^{\circ} \mathrm{C}$. A gas chromatography system traps and separates the detected gases followed by detection by a TCD (thermal conductivity detector), with $\mathrm{C}$ determined as $\mathrm{CO}_{2}$ and $\mathrm{N}$ as $\mathrm{N}_{2}$.

For S determinations, solid samples were loaded into tin capsules, and weighed out on a Mettler Toledo XP2U microbalance with elemental compositions determined using an Elementar Vario PyroCube EA. Samples dropped into the EA were combusted at $1020^{\circ}$ $\mathrm{C}$ over tungsten oxide in a continuous stream of helium carrier gas to produce $\mathrm{SO}_{3}$ from any sulfur present in the sample. The resulting gas then passed through a reduced copper reactor at $650^{\circ} \mathrm{C}$ to reduce $\mathrm{SO}_{3}$ to $\mathrm{SO}_{2}$, and trap any volatile halogen compounds on silver wool. Following water removal using a Sicapent adsorption tube, the $\mathrm{SO}_{2}$ analyte gases were separated and purified using purge-trap columns, and then carried through a 
thermal conductivity detector (TCD). The TCD signal is passed to computer software that calculates elemental abundances based on integrated TCD peak areas.

For S analyses, we ran samples A1 and B1 once, and sample $\mathrm{C} 1$ in duplicate as a check for consistency. $\mathrm{C}$ was also determined as $\mathrm{CO}_{2}$ by this method. This yielded results entirely consistent with those from the above $\mathrm{CN}$ analyses, but with larger errors resulting from the smaller sample size, and is thus not reported, here. Lower limits for quantification and errors were determined by running 3 concentration standards containing the elements of interest and 3 blank samples will be run prior to analyzing samples.

To measure oxygen concentrations, powdered samples were loaded into Ni crucibles. This procedure then uses a reaction with chlorine trifluoride $\left(\mathrm{ClF}_{3}\right)$ to convert oxygen from silicate and oxide materials into oxygen gas $\left(\mathrm{O}_{2}\right)$. The $\mathrm{O}_{2}$ is subsequently converted to carbon dioxide gas $\left(\mathrm{CO}_{2}\right)$, and yield is measured using a manometer. The use of high vacuum fluorination systems to extract oxygen from silicate and other oxide phases using halogen fluorides or fluorine gas is well documented in peer-reviewed literature. The typical uncertainty for runs in this laboratory is quoted here $( \pm 0.2 \%)$.

Table 4: Organic Composition Results

\begin{tabular}{c|c|c|c|c|c|c|c} 
Sample & \multicolumn{1}{c}{$\% \mathrm{~N}$} & \multicolumn{1}{c}{$1 \mathrm{sd}$} & \multicolumn{1}{c}{$\% \mathrm{C}$} & \multicolumn{1}{c}{$\% \mathrm{O} d$} & $\% \mathrm{~S}$ \\
\hline $\mathrm{A} 1$ & 0.0126 & 0.0005 & 0.148 & 0.011 & 51.77 & 0.20 & $<0.06$ \\
\hline $\mathrm{B} 1$ & 0.0189 & 0.0126 & 0.294 & 0.027 & 45.63 & 0.20 & $<0.06$ \\
\hline $\mathrm{C} 1$ & 0.0041 & 0.0005 & 0.220 & 0.033 & 47.74 & 0.20 & $<0.06$
\end{tabular}

Values are given as an elemental percent. Errors are the standard deviation from the triplicate analyses for $\mathrm{C}$ and $\mathrm{N}$, where variance for all samples and analyses was $0.1 \%$ or less. O errors are typical uncertainties. $\mathrm{S}$ concentrations were below detection limits $(0.0006 \mathrm{~g} / \mathrm{g})$ for all samples.

\section{Non-Destructive Major and Trace Element Analyses}

Aliquots of powders from each of the three samples were analyzed by X-ray fluorescence (XRF) spectrometry, which is used for routine, relatively non-destructive chemical analyses of rocks, minerals, sediments and fluids. XRF bombards the sample with highenergy X-rays, causing the emission of characteristic "secondary" (or fluorescent) X-rays from elements higher in atomic number than Be (although, in practice, only X-rays from elements higher in atomic number than $\mathrm{Na}$ are detected). XRF often provides complementary information to that provided by ICP-MS (see ICP-MS data, below and Tables 7 and 8).

Whole-rock samples were analyzed for element concentrations using a Bruker S8 WDXRF. Analyses were performed on powdered samples in a helium atmosphere using the Bruker QuantExpress analytical program. The instrument calibration was performed by measuring standard silicate glass discs (Breitländer $\mathrm{GmbH}$ ), certified for a suite of major and trace elements, under the same operating conditions as the samples. All elements from $\mathrm{Na}$ to $\mathrm{U}$ were analyzed; only elements measured above detection limits (Table 5) 
Table 5: XRF trace and major element results

\begin{tabular}{|c|c|c|c|c|c|c|c|c|c|c|}
\hline Oxide & $\mathrm{Z}$ & A1 & $1 \sigma(\%)$ & $\begin{array}{c}\text { LLD } \\
\text { (ppm) }\end{array}$ & B1 & $1 \sigma(\%)$ & $\begin{array}{c}\text { LLD } \\
(\mathrm{ppm})\end{array}$ & $\mathrm{C} 1$ & $1 \sigma(\%)$ & $\begin{array}{c}\text { LLD } \\
\text { (ppm) }\end{array}$ \\
\hline $\mathrm{Na}_{2} \mathrm{O}$ & 11 & $1.14 \%$ & 4.47 & 300.1 & $1.05 \%$ & 4.91 & 330.0 & $1.33 \%$ & 4.18 & 297.2 \\
\hline $\mathrm{MgO}$ & 12 & $1.59 \%$ & 1.75 & 198.9 & $1.63 \%$ & 1.75 & 193.3 & $0.96 \%$ & 2.39 & 187.7 \\
\hline $\mathrm{Al}_{2} \mathrm{O}_{3}$ & 13 & $13.25 \%$ & 0.52 & 118.8 & $12.80 \%$ & 0.54 & 115.4 & $12.05 \%$ & 0.56 & 112.9 \\
\hline $\mathrm{SiO}_{2}$ & 14 & $67.90 \%$ & 0.21 & 133.5 & $66.23 \%$ & 0.22 & 125.0 & $68.24 \%$ & 0.22 & 135.2 \\
\hline $\mathrm{P}_{2} \mathrm{O}_{5}$ & 15 & $0.23 \%$ & 4.32 & 109.6 & $0.21 \%$ & 4.60 & 104.9 & $0.16 \%$ & 5.37 & 100.2 \\
\hline $\mathrm{SO}_{3}$ & 16 & $0.06 \%$ & 7.72 & 74.1 & $0.06 \%$ & 7.54 & 73.2 & $0.04 \%$ & 9.20 & 71.7 \\
\hline $\mathrm{Cl}$ & 17 & $0.03 \%$ & 9.09 & 53.6 & nd & 25.90 & 50.1 & nd & 23.90 & 51.5 \\
\hline $\mathrm{K}_{2} \mathrm{O}$ & 19 & $6.18 \%$ & 0.34 & 40.2 & $6.60 \%$ & 0.33 & 39.0 & $7.25 \%$ & 0.32 & 41.2 \\
\hline $\mathrm{CaO}$ & 20 & $4.29 \%$ & 0.44 & 92.0 & $6.08 \%$ & 0.38 & 96.1 & $6.30 \%$ & 0.38 & 101.1 \\
\hline $\mathrm{TiO}_{2}$ & 22 & $0.71 \%$ & 1.06 & 55.1 & $0.73 \%$ & 1.09 & 57.2 & $0.47 \%$ & 1.39 & 57.7 \\
\hline $\mathrm{Cr}_{2} \mathrm{O}_{3}$ & 24 & $68 \mathrm{ppm}$ & 19.00 & 32.5 & nd & 71.70 & 34.2 & nd & 42.50 & 34.2 \\
\hline $\mathrm{MnO}$ & 25 & $0.14 \%$ & 1.35 & 25.5 & $0.14 \%$ & 1.41 & 24.7 & $0.13 \%$ & 1.51 & 24.2 \\
\hline $\mathrm{Fe}_{2} \mathrm{O}_{3}$ & 26 & $4.18 \%$ & 0.20 & 82.2 & $4.19 \%$ & 0.20 & 83.9 & $2.82 \%$ & 0.25 & 22.8 \\
\hline $\mathrm{NiO}$ & 28 & nd & 34.30 & 15.4 & $54 \mathrm{ppm}$ & 11.30 & 16.0 & $39 \mathrm{ppm}$ & 15.30 & 15.1 \\
\hline $\mathrm{CuO}$ & 29 & $59 \mathrm{ppm}$ & 8.80 & 13.1 & $50 \mathrm{ppm}$ & 10.80 & 13.4 & $53 \mathrm{ppm}$ & 10.10 & 13.2 \\
\hline $\mathrm{ZnO}$ & 30 & $0.01 \%$ & 3.45 & 11.6 & $0.02 \%$ & 3.36 & 11.9 & $0.01 \%$ & 4.40 & 11.6 \\
\hline $\mathrm{Ga}_{2} \mathrm{O}_{3}$ & 31 & $43 \mathrm{ppm}$ & 10.10 & 12.5 & $40 \mathrm{ppm}$ & 11.50 & 12.9 & $51 \mathrm{ppm}$ & 9.12 & 12.1 \\
\hline $\mathrm{As}_{2} \mathrm{O}_{3}$ & 33 & nd & 9.70 & 16.1 & nd & 10.30 & 16.5 & $47 \mathrm{ppm}$ & 8.71 & 10.3 \\
\hline $\mathrm{Rb}_{2} \mathrm{O}$ & 37 & $0.03 \%$ & 1.04 & 8.0 & $0.03 \%$ & 1.11 & 8.2 & $0.04 \%$ & 1.00 & 8.0 \\
\hline $\mathrm{SrO}$ & 38 & $0.05 \%$ & 0.81 & 7.1 & $0.05 \%$ & 0.87 & 7.4 & $0.03 \%$ & 1.18 & 7.3 \\
\hline $\mathrm{Y}_{2} \mathrm{O}_{3}$ & 39 & $5 \mathrm{ppm}$ & 2.60 & 14.8 & $10 \mathrm{ppm}$ & 2.65 & 15.2 & nd & 2.57 & 15.6 \\
\hline $\mathrm{ZrO}_{2}$ & 40 & $0.08 \%$ & 0.76 & 14.8 & $0.08 \%$ & 0.84 & 15.2 & $0.07 \%$ & 1.00 & 13.8 \\
\hline $\mathrm{Nb}_{2} \mathrm{O}_{5}$ & 41 & $50 \mathrm{ppm}$ & 6.27 & 9.4 & $36 \mathrm{ppm}$ & 8.85 & 9.9 & $44 \mathrm{ppm}$ & 7.71 & 9.6 \\
\hline $\mathrm{BaO}$ & 56 & $0.09 \%$ & 5.79 & 176.5 & $0.10 \%$ & 5.46 & 182.9 & $0.08 \%$ & 6.72 & 172.2 \\
\hline $\mathrm{CeO}_{2}$ & 58 & $0.02 \%$ & 7.83 & 273.5 & nd & 22.00 & 290.0 & nd & 12.20 & 280.2 \\
\hline $\mathrm{PbO}$ & 82 & $51 \mathrm{ppm}$ & 15.80 & 21.7 & 48 ppm & 17.80 & 22.3 & nd & 54.40 & 22.4 \\
\hline
\end{tabular}

Sample concentrations are given as weight percent oxides (\%), or as parts-per-million by weight (ppm). To convert, for example, $0.09 \%=900 \mathrm{ppm}$, in these units. The lower limits of detection (LLD) are calculated for each sample in ppm. A list of oxides that were not detected in any of the three samples, and their detection limits, is provided in Table 6. 
Table 6: Elements and oxides not detected by XRF

\begin{tabular}{|c|c|c|}
\hline $\begin{array}{c}\text { Element } \\
\text { or Oxide }\end{array}$ & $\mathrm{Z}$ & $\begin{array}{c}\text { Average } \\
\text { LLD } \\
\text { (ppm) }\end{array}$ \\
\hline $\mathrm{Sc}_{2} \mathrm{O}_{3}$ & 21 & 49.5 \\
\hline $\mathrm{V}_{2} \mathrm{O}_{5}$ & 23 & 84.6 \\
\hline $\mathrm{CoO}$ & 27 & 33.2 \\
\hline $\mathrm{GeO}_{2}$ & 32 & 11.2 \\
\hline $\mathrm{SeO}_{2}$ & 34 & 17.5 \\
\hline $\mathrm{Br}$ & 35 & 9.1 \\
\hline $\mathrm{MoO}_{3}$ & 42 & 13.5 \\
\hline $\mathrm{Ru}$ & 44 & 17.5 \\
\hline $\mathrm{Rh}$ & 45 & 18.4 \\
\hline $\mathrm{Pd}$ & 46 & 19.7 \\
\hline $\mathrm{Ag}$ & 47 & 19.9 \\
\hline $\mathrm{CdO}_{2}$ & 48 & 23.4 \\
\hline $\mathrm{In}_{2} \mathrm{O}_{3}$ & 49 & 27.2 \\
\hline $\mathrm{SnO}_{2}$ & 50 & 38.3 \\
\hline $\mathrm{Sb}_{2} \mathrm{O}_{3}$ & 51 & 38.9 \\
\hline $\mathrm{TeO}_{2}$ & 52 & 65.3 \\
\hline $\mathrm{I}$ & 53 & 58.8 \\
\hline $\mathrm{Cs}_{2} \mathrm{O}$ & 55 & 92.5 \\
\hline $\mathrm{La}_{2} \mathrm{O}_{3}$ & 57 & 110.2 \\
\hline $\mathrm{Pr}_{6} \mathrm{O}_{11}$ & 59 & 96.4 \\
\hline $\mathrm{Nd}_{2} \mathrm{O}_{3}$ & 60 & 112.5 \\
\hline
\end{tabular}

\begin{tabular}{|c|c|c|}
\hline $\begin{array}{l}\text { Element } \\
\text { or Oxide }\end{array}$ & $\mathrm{Z}$ & $\begin{array}{c}\text { Average } \\
\text { LLD } \\
(\mathrm{ppm})\end{array}$ \\
\hline $\mathrm{Sm}_{2} \mathrm{O}_{3}$ & 62 & 88.5 \\
\hline $\mathbf{E u}_{2} \mathbf{O}_{3}$ & 63 & 141.0 \\
\hline $\mathbf{G d}_{2} \mathrm{O}_{3}$ & 64 & 70.1 \\
\hline $\mathbf{T b}_{4} \mathbf{O}_{7}$ & 65 & 121.2 \\
\hline $\mathrm{Dy}_{2} \mathrm{O}_{3}$ & 66 & 154.3 \\
\hline $\mathrm{Ho}_{2} \mathrm{O}_{3}$ & 67 & 49.1 \\
\hline $\mathrm{Er}_{2} \mathrm{O}_{3}$ & 68 & 45.9 \\
\hline $\mathbf{Y} \mathbf{b}_{2} \mathbf{O}_{3}$ & 70 & 59.3 \\
\hline $\mathrm{HfO}_{2}$ & 72 & 51.4 \\
\hline $\mathrm{Ta}_{2} \mathrm{O}_{5}$ & 73 & 41.0 \\
\hline $\mathrm{WO}_{3}$ & 74 & 38.0 \\
\hline $\mathbf{R e}$ & 75 & 69.7 \\
\hline Ir & 77 & 31.9 \\
\hline $\mathbf{P t}$ & 78 & 25.2 \\
\hline Au & 79 & 23.8 \\
\hline Hg & 80 & 23.1 \\
\hline Tl & 81 & 31.7 \\
\hline $\mathbf{B i}_{2} \mathbf{O}_{3}$ & 83 & 20.0 \\
\hline $\mathrm{ThO}_{2}$ & 90 & 26.5 \\
\hline $\mathbf{U O}_{2}$ & 92 & 23.4 \\
\hline
\end{tabular}

Numbers represent the average lower detection limit for each element or oxide species as calculated as the average lower limit of detection (LLD) for each sample calculated as parts-per-million by weight (ppm).

are reported. Table 6 lists elements analyzed for but not detected above detection limits, which were typically on the order of 50-200 ppm. Due to the fact that samples were analyzed non-destructively as loose powders, typical analytical sum totals were $\sim 50 \%$. Results are reported as oxides, assuming stoichiometric relationships, normalized to $100 \%$ totals.

\section{Destructive Major and Trace Element Analyses}

Aliquots of the solution intended for trace elemental analysis were diluted in a $\mathrm{HNO}_{3} / \mathrm{HF}$ solution to elemental concentrations of $\sim 200 \mathrm{ppm}$ and were then analyzed on a Thermo Electron X7 Quadrupole Inductively Coupled Mass Spectrometer. An internal standard corrects for instrument drift and suppression from the matrix. The results are summarized in the Tables 7 and 8. Major element concentrations are given as elemental weight percent. Trace element concentrations are shown in parts-per-million by weight 
(micrograms/gram of sample) along with the calculated uncertainties. Masses were calculated from powders as received, with no additional drying.

Table 7: ICP-MS major element results

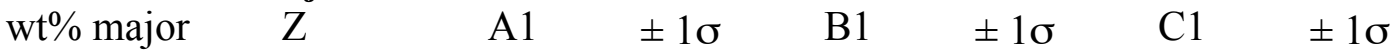
element

\begin{tabular}{c|l|l|l|l|l|l|l}
\hline $\mathrm{Na}$ & 11 & 1.856 & 0.081 & 1.858 & 0.068 & 2.128 & 0.106 \\
\hline $\mathrm{Mg}$ & 12 & 0.611 & 0.014 & 0.508 & 0.012 & 0.301 & 0.013 \\
\hline $\mathrm{Al}$ & 13 & 6.048 & 0.111 & 5.821 & 0.136 & 5.696 & 0.180 \\
\hline $\mathrm{K}$ & 19 & 2.904 & 0.032 & 3.032 & 0.111 & 3.263 & 0.092 \\
\hline $\mathrm{Ca}$ & 20 & 1.253 & 0.024 & 1.490 & 0.047 & 1.254 & 0.029 \\
\hline $\mathrm{Ti}$ & 22 & 0.186 & 0.002 & 0.154 & 0.003 & 0.099 & 0.003 \\
\hline $\mathrm{Cr}$ & 24 & 0.002 & 0.000 & 0.002 & 0.000 & 0.001 & 0.000 \\
\hline $\mathrm{Mn}$ & 25 & 0.052 & 0.000 & 0.048 & 0.001 & 0.042 & 0.001 \\
\hline $\mathrm{Fe}$ & 26 & 1.337 & 0.027 & 1.118 & 0.022 & 0.755 & 0.016
\end{tabular}

All three samples show very similar major element chemistry, with sample A1 and B1 more closely overlapping than sample $\mathrm{C} 1$. The largest major element variations are in the elemental iron content of the samples $(\sim 50 \%)$. Si (calculated as $\left.\mathrm{SiO}_{2}\right)$ variations are comparatively minor (see XRF data, Table 5). 51 trace elements were also detected above backgrounds. Trace element data also yield extremely similar chemical compositions, with sample A1 and B1 more closely overlapping than sample $\mathrm{C} 1$.

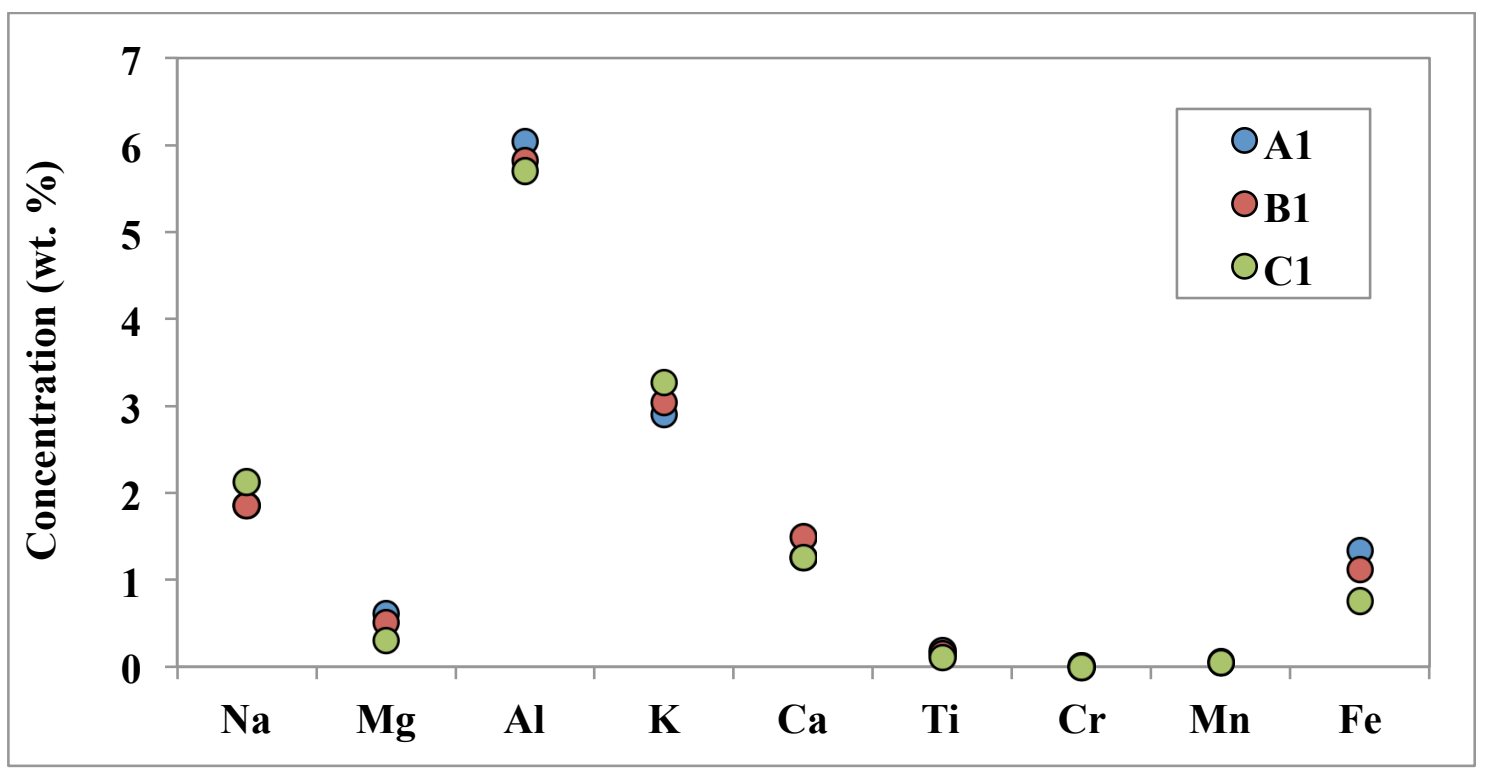

Figure 3: The major element concentrations for the three soil samples. Error bars are smaller than the symbols. 
Table 8: ICP-MS trace element results

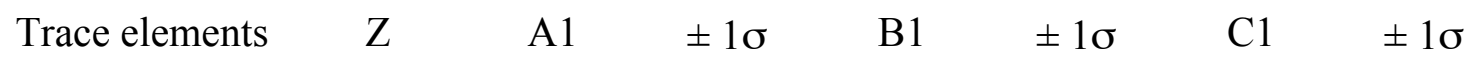
ppm-w

\begin{tabular}{|c|c|c|c|c|c|c|c|}
\hline $\mathrm{Be}$ & 4 & 2.819 & 0.192 & 2.818 & 0.106 & 2.926 & 0.301 \\
\hline $\mathrm{V}$ & 23 & 98.112 & 5.860 & 71.957 & 1.594 & 56.282 & 1.382 \\
\hline Co & 27 & 3.459 & 0.090 & 2.689 & 0.084 & 1.227 & 0.055 \\
\hline $\mathrm{Ni}$ & 28 & 6.753 & 0.349 & 5.033 & 0.207 & 2.161 & 0.189 \\
\hline $\mathrm{Cu}$ & 29 & 7.085 & 0.203 & 5.457 & 0.126 & 2.782 & 0.126 \\
\hline $\mathrm{Zn}$ & 30 & 50.691 & 1.485 & 45.965 & 2.054 & 36.694 & 0.804 \\
\hline $\mathrm{Ga}$ & 31 & 15.977 & 0.248 & 15.038 & 0.472 & 14.338 & 0.271 \\
\hline $\mathrm{Ge}$ & 32 & 8.298 & 1.116 & 6.400 & 0.839 & 5.676 & 0.781 \\
\hline As & 33 & 5.337 & 0.475 & 4.975 & 0.445 & 4.099 & 0.457 \\
\hline $\mathrm{Se}$ & 34 & 0.633 & 0.143 & 0.709 & 0.188 & 0.483 & 0.122 \\
\hline $\mathrm{Rb}$ & 37 & 137.300 & 1.521 & 131.358 & 3.855 & 142.766 & 3.057 \\
\hline $\mathrm{Sr}$ & 38 & 187.787 & 1.209 & 166.179 & 4.259 & 96.589 & 1.535 \\
\hline $\mathrm{Y}$ & 39 & 21.580 & 0.426 & 24.646 & 0.831 & 21.542 & 0.416 \\
\hline $\mathrm{Zr}$ & 40 & 71.542 & 0.795 & 69.320 & 5.265 & 70.603 & 6.479 \\
\hline $\mathrm{Nb}$ & 41 & 18.751 & 0.230 & 18.518 & 0.479 & 18.064 & 0.444 \\
\hline Mo & 42 & 1.264 & 0.085 & 1.063 & 0.100 & 1.124 & 0.073 \\
\hline $\mathrm{Ru}$ & 44 & 0.002 & 0.003 & 0.001 & 0.001 & 0.001 & 0.003 \\
\hline $\mathrm{Rh}$ & 45 & 0.003 & 0.002 & 0.002 & 0.001 & 0.001 & 0.001 \\
\hline $\mathrm{Pd}$ & 46 & 0.513 & 0.039 & 0.589 & 0.073 & 0.501 & 0.056 \\
\hline $\mathrm{Ag}$ & 47 & 0.219 & 0.033 & 0.194 & 0.013 & 0.188 & 0.016 \\
\hline $\mathrm{Cd}$ & 48 & 0.130 & 0.031 & 0.127 & 0.019 & 0.085 & 0.018 \\
\hline $\mathrm{Sn}$ & 50 & 1.827 & 0.054 & 1.775 & 0.059 & 1.799 & 0.236 \\
\hline $\mathrm{Sb}$ & 51 & 0.584 & 0.045 & 0.486 & 0.028 & 0.426 & 0.030 \\
\hline $\mathrm{Te}$ & 52 & 0.034 & 0.065 & 0.011 & 0.014 & 0.016 & 0.029 \\
\hline Cs & 55 & 3.662 & 0.080 & 3.216 & 0.054 & 2.939 & 0.051 \\
\hline $\mathrm{Ba}$ & 56 & 367.505 & 5.933 & 327.415 & 8.594 & 218.961 & 3.672 \\
\hline $\mathrm{La}$ & 57 & 36.659 & 0.617 & 35.706 & 0.689 & 35.264 & 0.663 \\
\hline $\mathrm{Ce}$ & 58 & 71.480 & 1.948 & 70.414 & 1.531 & 69.613 & 1.687 \\
\hline $\operatorname{Pr}$ & 59 & 8.275 & 0.082 & 8.220 & 0.335 & 7.934 & 0.203 \\
\hline $\mathrm{Nd}$ & 60 & 28.976 & 0.152 & 28.780 & 0.982 & 27.388 & 0.617 \\
\hline $\mathrm{Sm}$ & 62 & 5.226 & 0.081 & 5.354 & 0.351 & 5.028 & 0.115 \\
\hline $\mathrm{Eu}$ & 63 & 0.702 & 0.025 & 0.684 & 0.012 & 0.516 & 0.021 \\
\hline $\mathrm{Gd}$ & 64 & 4.869 & 0.081 & 5.094 & 0.258 & 4.702 & 0.181 \\
\hline $\mathrm{Tb}$ & 65 & 0.694 & 0.030 & 0.740 & 0.025 & 0.683 & 0.028 \\
\hline Dy & 66 & 3.994 & 0.148 & 4.401 & 0.132 & 3.943 & 0.038 \\
\hline Ho & 67 & 0.760 & 0.015 & 0.857 & 0.046 & 0.765 & 0.016 \\
\hline Er & 68 & 2.351 & 0.142 & 2.605 & 0.057 & 2.302 & 0.050 \\
\hline $\mathrm{Tm}$ & 69 & 0.332 & 0.022 & 0.366 & 0.012 & 0.332 & 0.018 \\
\hline $\mathrm{Yb}$ & 70 & 2.242 & 0.034 & 2.441 & 0.065 & 2.241 & 0.051 \\
\hline
\end{tabular}


Table 8: ICP-MS trace element results, continued

\begin{tabular}{|c|c|c|c|c|c|c|c|}
\hline $\begin{array}{l}\text { Trace elements } \\
\text { ppm-w }\end{array}$ & Z & A1 & $\pm 1 \sigma$ & B1 & $\pm 1 \sigma$ & $\mathrm{C} 1$ & $\pm 1 \sigma$ \\
\hline $\mathrm{Lu}$ & 71 & 0.323 & 0.008 & 0.355 & 0.019 & 0.321 & 0.013 \\
\hline Hf & 72 & 2.822 & 0.154 & 2.635 & 0.127 & 2.666 & 0.145 \\
\hline $\mathrm{Ta}$ & 73 & 1.802 & 0.105 & 1.739 & 0.147 & 1.841 & 0.160 \\
\hline W & 74 & 1.525 & 0.059 & 1.439 & 0.068 & 1.293 & 0.043 \\
\hline $\mathrm{Re}$ & 75 & 0.003 & 0.004 & 0.003 & 0.001 & 0.003 & 0.001 \\
\hline Ir & 77 & 0.011 & 0.004 & 0.011 & 0.004 & 0.011 & 0.003 \\
\hline $\mathrm{Pt}$ & 78 & 0.027 & 0.003 & 0.029 & 0.008 & 0.029 & 0.012 \\
\hline $\mathrm{Au}$ & 79 & 0.240 & 0.040 & 0.191 & 0.022 & 0.176 & 0.018 \\
\hline $\mathrm{Tl}$ & 81 & 0.794 & 0.029 & 0.741 & 0.031 & 0.795 & 0.031 \\
\hline $\mathrm{Pb}$ & 82 & 22.254 & 0.162 & 21.738 & 0.781 & 20.872 & 0.150 \\
\hline $\mathrm{Th}$ & 90 & 15.469 & 0.436 & 15.893 & 0.775 & 16.935 & 0.618 \\
\hline $\mathrm{U}$ & 92 & 3.169 & 0.044 & 3.238 & 0.113 & 3.188 & 0.040 \\
\hline
\end{tabular}

\section{Summary}

All three samples, while quite distinct in character from other locations at the National Nuclear Security Site, are similar to one another even in the case of the roadbed materials. This suggests that the roadbed material was derived nearby, and does not significantly alter the chemical makeup of the surrounding area. There may be minor differences in density between the nearby soils compared with the roadbed, however, depending on how the road was constructed.

The total water content (not measured here), and corresponding $\mathrm{H}$ and $\mathrm{O}$ concentrations, will likely vary with season due the presence of clays and weathered minerals in these soils. Composition-based calculations based on these data might consider changing the concentration of $\mathrm{H}_{2} \mathrm{O}$ from 0.2 to $5.0 \%$ by weight (a reasonable range of water contents), to understand the importance of this effect.

Where non-destructive XRF and dissolution-based ICP-MS overlap, reporting measurements of the same elements, the overlap is in good agreement. For calculation purposes, however, ICP-MS data are to be preferred, as they are based on assumptions of normal isotopic abundances, rather than stoichiometric relationships, and tend to be better quantified (smaller and better characterized errors). 


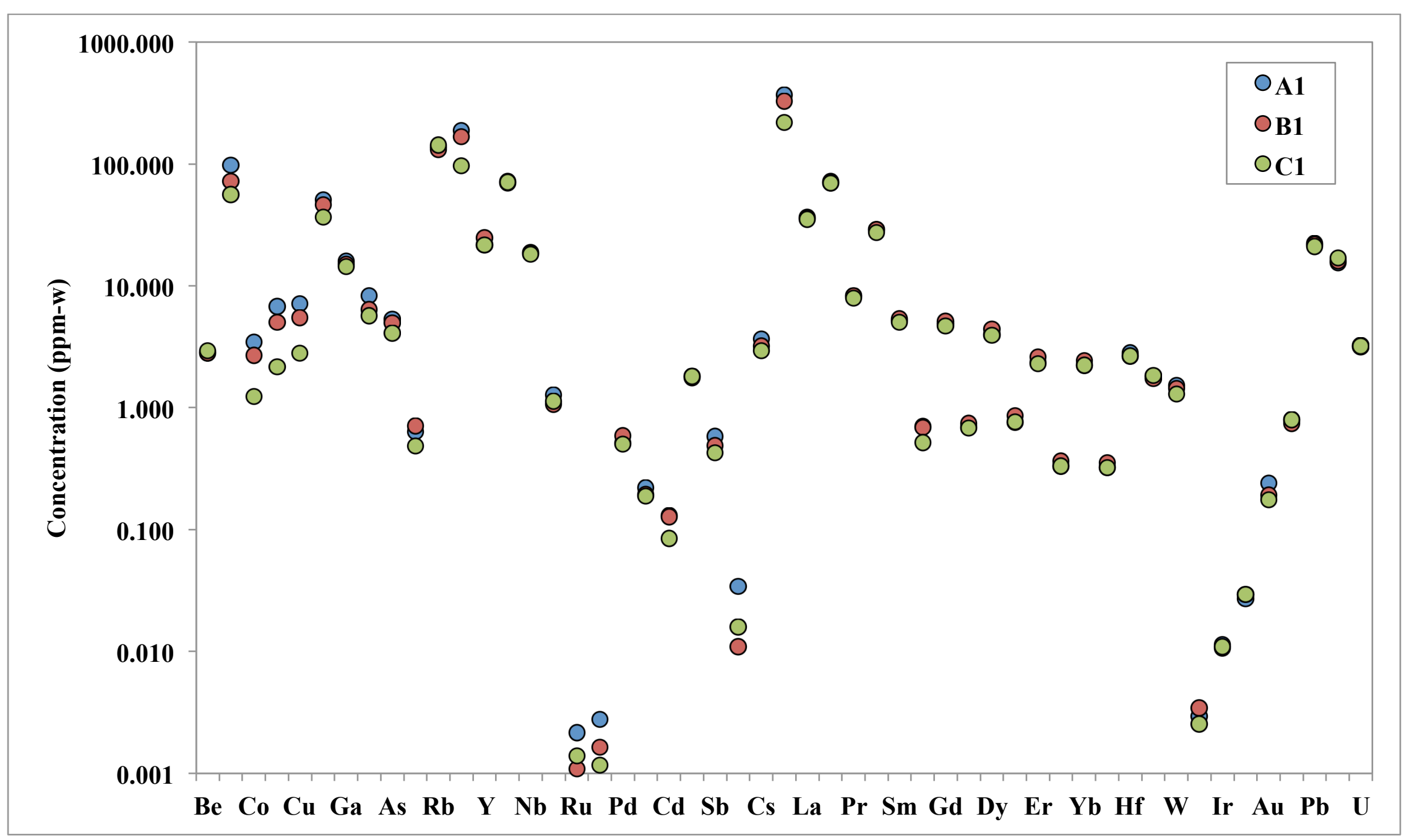

Figure 4: Trace element concentrations for the three soil samples. Error bars are smaller than the symbols. 